\title{
A Data-Driven Hybrid Three-Stage Framework for Hospital Bed Allocation: A Case Study in a Large Tertiary Hospital in China
}

\author{
Li Luo $\mathbb{D}$, ${ }^{1}$ Jialing Li $\mathbb{D},{ }^{1}$ Xueru Xu $\mathbb{D},{ }^{1}$ Wenwu Shen $\mathbb{D},{ }^{2}$ and Lin Xiao $\mathbb{D}{ }^{1}$ \\ ${ }^{1}$ Business School of Sichuan University, No. 24 South Section 1, Yihuan Road, Chengdu, China \\ ${ }^{2}$ West China Hospital of Sichuan University, No. 17 People's South Road, Chengdu, China \\ Correspondence should be addressed to Xueru Xu; 18200291987@163.com and Wenwu Shen; wenwu_shen@163.com
}

Received 24 December 2018; Revised 6 March 2019; Accepted 2 April 2019; Published 2 May 2019

Guest Editor: Giedrius Vanagas

Copyright (c) 2019 Li Luo et al. This is an open access article distributed under the Creative Commons Attribution License, which permits unrestricted use, distribution, and reproduction in any medium, provided the original work is properly cited.

Beds are key, scarce medical resources in hospitals. The bed occupancy rate (BOR) amongst different departments within large tertiary hospitals is very imbalanced, a situation which has led to problems between the supply of and the demand for bed resources. This study aims to balance the utilization of existing beds in a large tertiary hospital in China. We developed a datadriven hybrid three-stage framework incorporating data analysis, simulation, and mixed integer programming to minimize the gaps in BOR among different departments. The first stage is to calculate the length of stay (LOS) and BOR of each department and identify the departments that need to be allocated beds. In the second stage, we used a fitted arrival distribution and median LOS as the input to a generic simulation model. In the third stage, we built a mixed integer programming model using the results obtained in the first two stages to generate the optimal bed allocation strategy for different departments. The value of the objective function, $Z$, represents the severity of the imbalance in BOR. Our case study demonstrated the effectiveness of the proposed data-driven hybrid three-stage framework. The results show that $Z$ decreases from 0.7344 to 0.0409 after re-allocation, which means that the internal imbalance has eased. Our framework provides hospital bed policy makers with a feasible solution for bed allocation.

\section{Introduction}

The inherent difference between limited resources for healthcare and steadily increasing demands occurs all over the world and is particularly serious in developing countries. According to a research report from the World Health Organization (WHO) and World Bank Groups, at least 400 million people worldwide cannot receive one or more basic health services [1].

This differential is particularly apparent with respect to bed resources. Although hospital bed numbers have increased greatly in recent years, this increase cannot cope with the growth rate of admission demand in China. According to the report "Statistical Communique on the Development of China's Health and Family Planning Program 2016" [2], the number of hospitalizations across the nation's medical and health institutions was 227.28 million and the annual hospitalization rate was $16.5 \%$. There were 7.410 million beds in medical institutions across the country.
Amongst all of the medical institutions, China's large tertiary hospitals, classed as Class III according to the classification standards (Appendix), are facing the most serious imbalance between admissions and bed resources (Table 1). The number of hospital beds and individuals hospitalized in Class III hospitals increased to 2,213,718 and 76,860,000, respectively. Bed occupancy rates (BORs) reached $98.8 \%$ in 2016.

We found that the imbalance between supply and demand in large tertiary (Class III) hospitals is greater than that in the other two classes of hospitals. Hospital managers urgently need to find solutions to alleviate bed shortages. Hospital administrators typically address this issue in two ways: by improving utilization of existing beds or by expanding capacity. The first involves the complex task of strategically allocating the proper amount of beds for each set of care types. A hospital that is unable to find an optimal allocation may acquire additional beds. However, an expansion, which is desirable for one hospital, may not be 
TABLE 1: Hospital beds, hospitalization, and BOR of China's large tertiary hospitals in 2015 and 2016.

\begin{tabular}{lcccccc}
\hline \multirow{2}{*}{ Hospital level } & \multicolumn{2}{c}{ Hospital beds } & \multicolumn{2}{c}{ Hospitalization } & \multicolumn{2}{c}{ BOR } \\
& 2015 & 2016 & 2015 & 2016 & $2015(\%)$ & $2016(\%)$ \\
\hline Class III & $2,047,819$ & $2,213,718$ & $68,290,000$ & $76,860,000$ & 98.80 & 98.80 \\
Class II & $2,196,748$ & $2,302,887$ & $71,210,000$ & $75,700,000$ & 84.10 & 84.20 \\
Class I & 481,876 & 517,837 & $9,650,000$ & $10,390,000$ & 58.80 & 58 \\
\hline
\end{tabular}

advantageous from the perspective of the public planner [3] and may even have some negative consequences, such as doctor and nurse work overloads, decreases in medical care quality, and aggravation of medical conflicts [4]. Therefore, from the perspective of the sustainability of medical resources, the best option is to improve the utilization of bed resources.

The BOR of the Class III hospitals is usually as high as $98 \%$. However, the utilization rate of beds in different departments of a Class III hospital can be very different. West China Hospital (WCH) is typical of such hospitals (see Section 3.1 for more details). The availability of beds for patient hospitalization services is excessive in some units and scarce in others. This discrepancy between bed availability in different departments leads to the overcrowding of some departments and the idleness of other departments. This internal imbalance has further worsened the shortage of hospital resources.

A possible solution to this problem is to allocate the number of beds among different departments in such a way as to increase the utilization rate of beds and alleviate the shortage of bed resources as much as possible. There has been considerable research into the allocation of bed resources. The study of bed resource allocation can be divided into two main approaches.

One approach is to assign the optimal number of beds in a single department or care units, such as surgery units [5], intensive care units [6,7], and obstetrics departments [8]. For instance, Akkerman and Knip [5] planned the optimal number of beds for cardiac surgery with the goal of reducing patient waiting time. Oerlemans et al. [9] sent an online questionnaire to all ICU physician members in 90 hospitals of the Dutch Society for Intensive Care, the results of which can be used to improve decision-making regarding allocation of ICU resources. Devapriya et al. [10] proposed the strategic bed analysis model, which is a discrete-event simulation model created after a thorough analysis of patient flow and data from Geisinger Health Systems (GHS). Ridge et al. [11] investigated the problem of hospital bed planning in the intensive care unit. Romanin-Jacur and Facchin [12] studied the ward planning of the intensive surgical department and the pediatric semiintensive care unit.

Another approach to the problem focuses on the number of hospital beds throughout the whole hospital. For example, Akcali et al. [13] planned the best use of hospital beds in the entire hospital with the goal of minimizing the total cost. Utley et al. [14] determined the reasonable number of beds for elective patients in the whole hospital in the case of a very low rate of patient cancellation. However, there is little research on the allocation of beds among different departments or wards.

A range of operational research (OR) methods have been developed and applied to problems of healthcare resource allocation [15], especially bed resources. These methods include queuing theory $[16,17]$, simulation $[18,19]$, goal programming $[20,21]$, and mathematical programming [22-24]. Most of the current research uses a single method for each study. However, the premise of queuing theory is based on very strong assumptions, and it is difficult to apply. Mathematical programming can flexibly add constraints and change the objective function according to specific conditions, and it is more popular. Therefore, a hybrid of different methods is more conducive to solving the problem. Simulation and mathematical programming have become increasingly popular approaches to allocate resources in health care. Tontarski [25] utilized simulation-based optimization and mathematical programming for solving complex nursescheduling problems. Studies on the bed allocation problem using mathematical programming, especially combined with simulation, are relatively few.

In short, research into bed allocation mostly focuses on the study of the number of beds in a single department. The problem of how to allocate beds in different departments has not been fully studied. Furthermore, most of the current research on bed allocation is based on a single method such as queuing theory [26] or simulation [27]. There are few hybrid models which integrate data analysis, simulation, and mathematical programming. We propose a solution to the bed allocation problem at an operational level. We develop a data-driven hybrid three-stage framework incorporating data analysis, simulation, and mixed integer programming (MIP) to determine the optimal bed allocation strategy. The first stage is to select departments for allocation according to the relationship between the number of beds and the BOR and have this result approved by hospital management. In the second stage, we used a simulation model to calculate the BOR for different numbers of hospital beds. We thus derive the functional relationship between the two variables. The third stage is to find the best number of beds in five departments using a MIP model. Our study aims to alleviate shortage of beds by balancing the utilization of existing beds without increasing the number of beds in a large hospital in China. Overall, the contributions of this study are as follows:

(1) Our framework is data driven, making the allocation strategy more rational. (i) Using real data from the hospital, data analysis is used to determine the optimal department needs to allocate beds; (ii) the simulation model is used to simulate the 
corresponding BOR under different bed allocation scenarios. The relationship between the hospital beds and the BOR is derived from the data, thereby providing data-driven personalized constraints for each department.

(2) The objective function of the MIP model is tailored to Chinese needs. Because the beds in large hospitals in China have been overloaded, we are not blindly reducing the BOR of beds but are keeping the number of beds in a reasonable range.

(3) This paper provides a general framework for the allocation of beds in the Chinese context. Different hospitals can modify the objective function of the model and appropriately add or reduce constraints according to their needs. Our model provides a reference for hospital management to effectively manage hospital bed resources.

The rest of the paper is organized as follows. In Section 2, we briefly introduced the background of the case hospital $(\mathrm{WCH})$ and the process of the data-driven hybrid threestage framework. Taking WCH as a case study, we applied the framework proposed in Section 2 to $\mathrm{WCH}$, and these analyses of the framework are shown in Section 3. In Section 4 , we discuss the results of the paper. Finally, Section 5 concludes the paper and indicates some directions for future research.

\section{Materials and Methods}

2.1. Study Hospital. From a macro perspective, the overall BOR in China's tertiary hospitals is very high. However, at an individual level, the availability of hospital beds in different departments in individual hospitals is uneven. This phenomenon exists in almost all of the tertiary hospitals in China. It is particularly serious in $\mathrm{WCH}$, a tertiary hospital which is located in Chengdu, Sichuan province. In order to rationally manage beds, the Admission Service Center (ASC), a bed planning organization, was established in 2011. It manages 2956 beds and 28 specialized care departments. After our survey and data analysis (Figure 1), we found that the allocation of beds among the 28 departments is not balanced. For example, the BOR of W3 is as high as $122 \%$, while others, such as $\mathrm{W} 12$, are only $64.70 \%$. The reason why the BOR is over $100 \%$ is that the extra beds are involved in the calculation process. When the number of inpatients exceeds the number of fixed beds, the hospital will add additional beds to meet the demand, and these beds are often arranged in the corridor. This imbalance further leads to inefficiency and waste of hospital bed space, which in turn exacerbates the shortage of hospital beds.

We focus on balancing the BOR of departments by redistributing beds to improve the utilization rate of resources. This study takes $\mathrm{WCH}$ as an example to provide a feasible solution for the shortage of hospital beds in large hospitals.

2.2. Data Collection. This study used data from the Hospital Information System of the ASC for the period from January
1 to December 31, 2013. It includes the time of each patient admission and discharge, demographic information, and department information and has a total of 243,685 admission registrations and 167,843 discharge records.

2.3. Methods. The aim of this research is to balance the BOR of each department by allocating the hospital beds to departments, using a fixed number of existing beds, and keeping the bed utilization rate of each department at a reasonable level. Hence, we proposed a data-driven hybrid three-stage framework to solve this problem. The overall approach is shown in Figure 2.

(1) Stage I (data preliminaries): we selected the key departments by analyzing their current BOR and number of beds.

(2) Stage II (construction of constraint conditions): Simio software [28] was used to establish a simulation model to obtain the different scenarios of the beds and corresponding $\mathrm{BOR}$. We then determined the relationship between the BOR and the number of beds through data fitting, which is one of the constraints of the Stage III.

(3) Stage III (construction of model): we established a MIP model to minimize the gap in BOR among different departments. We applied the genetic algorithm (GA) to solve this model, since GA is one of the best tools for satisfactory solution with advantages like good convergence, low computational complexity, high robustness, and so forth [29].

\subsubsection{Stage I: Data Preliminaries}

(1) Calculation of Length of Hospital Stay. Length of hospital stay (LOS) indicates the number of days the patient spent in a hospital bed. We made the assumption that the LOS can be considered as a constant [30]. We have got 243,685 admission records and 167,843 discharge records form ASC. The LOS is calculated as the discharge date for each patient minus the date of admission registration in the ASC. The sum of the days of all hospitalized patients is an important parameter for calculating the BOR. This paper uses the data from $20131 / 1$ to $2013 / 12 / 31$. We divided the patients into three types by the discharge date as follows and calculated the LOS in 2013 for each group.

(i) Type I: patients discharged during 2013. Those patients registered in the ASC during or before 2013. Their actual LOS during the 2013 is called LOS1. For example, the actual LOS of the patient who registered before 2013 is discharge date minus January 1, 2013.

(ii) Type II: those patients who left the hospital in 2014, who registered before 2013 or during 2013. Their LOS equals to December 1, 2013, minus registered date or December 31, 2013, minus January 1, 2013, which is named LOS2.

(iii) Type III: because some discharge data are missing, there are patients who are recorded as having been 


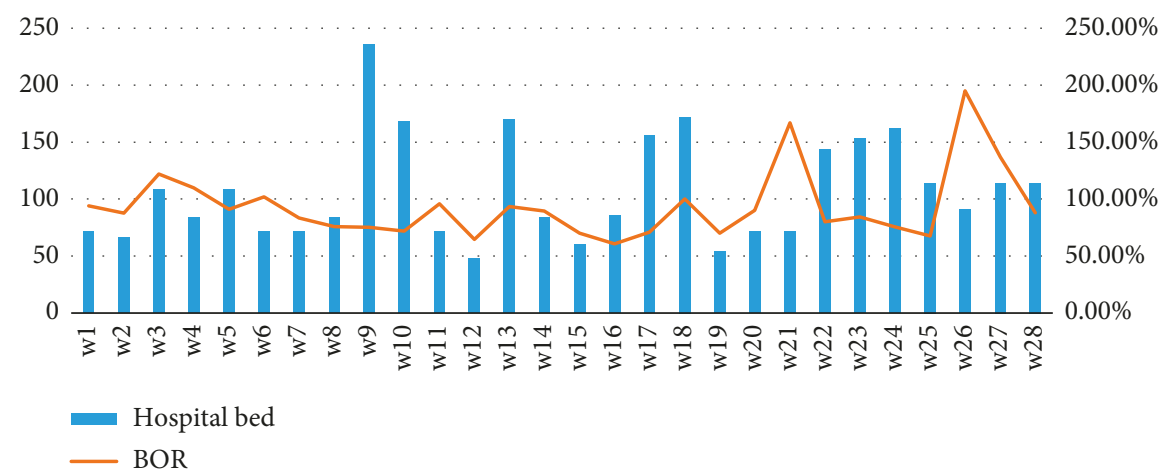

Figure 1: Hospital beds and BOR of 28 departments in West China Hospital.

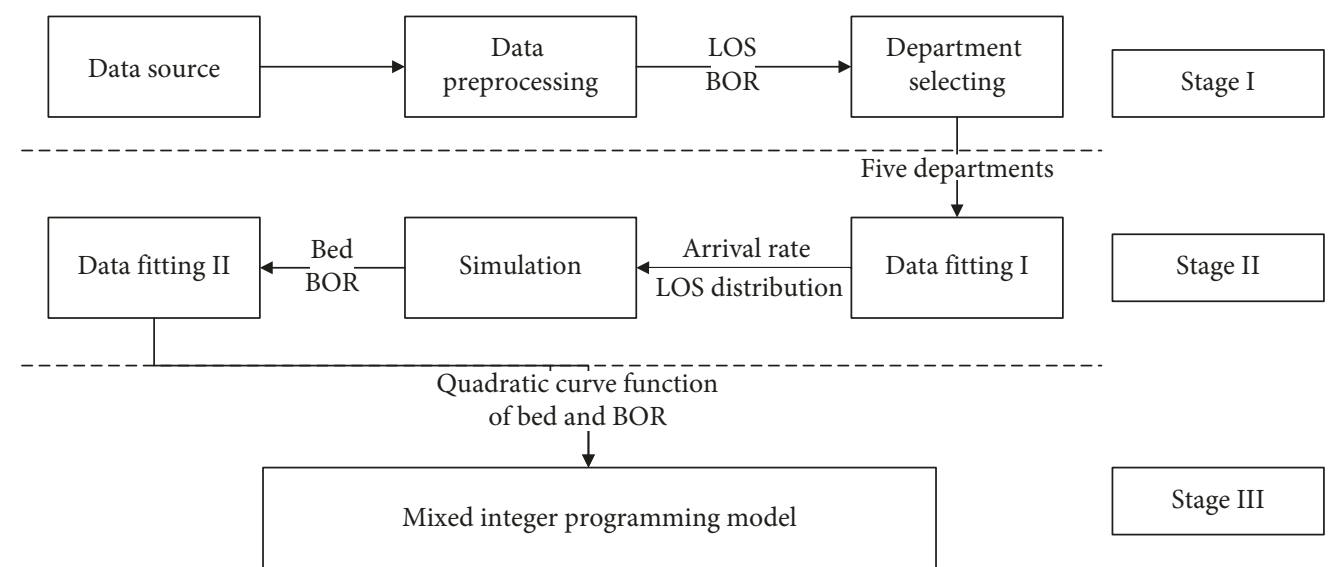

Figure 2: Methodology: process of the data-driven hybrid three-stage framework.

discharged from hospital in 2013 but had no admission records. These patients cannot be ignored. Hence, the number of Type III patients is the total number of discharged patients in one department in 2013 minus the number of Type I. We cannot directly calculate the LOS of Type III patients, so in this study, we used the median of the LOS of Type I patients as the value for the LOS of Type III patients, which we called LOS3.

(2) The Calculation of BOR. We calculated each department's BOR according to the following formulas:

$$
\text { all patients' LOS = LOS1 + LOS2 + LOS3, }
$$

$$
\text { BOR }=\frac{\text { all patients' LOS }}{\text { LOS that all beds can provide }}
$$

2.3.2. Stage II: Construction of Constraint Conditions. The Simio software was used to build simulation models using data from different departments. Changes in the number of beds, the number of hospitalized patients, and the LOS of patients with different bed numbers were simulated and used to calculate different BOR indexes. The relationship between hospital bed numbers and BOR was an important constraint condition in building the mixed integer programming model in Stage III. It proceeds in three steps:

(1) We used the EASY-FIT [31], a professional data fitting software, to fit the patient's arrival distribution and LOS distribution of each department

(2) The fitted arrival distribution and LOS distribution were used as the input to the simulation model, which identified the relationship between the number of beds and the BOR

(3) We fitted the relationship between the number of beds and the BOR via IBM SPSS Statistics V21 and obtained their functional relationship

2.3.3. Stage III: Construction of the Model. In order to thoroughly understand the hospital bed allocation problem, it is necessary to describe the characteristics of the problem in order to implement them in an appropriate mathematical model. We define parameters and variables of the model:

(1) Parameters

$K_{i j}$ : the ward type $j$ for department $i$. There are three inpatient ward types.

$K_{i 1}$ : the number of single-bed wards in department $i$. $K_{i 2}$ : the number of double-bed wards in department $i$. 
$K_{i 3}$ : the number of three-bed wards in department $i$

$L_{i}$ : lower bound of number of beds in department $i$.

$U_{i}$ : upper bound of number of beds in department $i$.

$C_{t}$ : the total number of beds of all the departments.

$\operatorname{BOR}_{i}\left(C_{i}\right)$ : the BOR of department $i$, when the number of beds of department $i$ is $C_{i}$. The relationship functions between $\mathrm{BOR}_{i}\left(C_{i}\right)$ and $C_{i}$ of department $i$ can be obtained from the results of Stage II. Here, we assume that the two variables are quadratic functions.

(2) Variable

$C_{i}$ : the number of beds of department $i$ and $C_{i}=K_{i 1}+2 K_{i 2}+3 K_{i 3} ; C_{i}$ is a positive integer.

There are $n$ departments in $\mathrm{WCH}$, but $i$ departments $(i \in 1,2, \ldots, n)$ need to be allocated beds. Our main decision variable is $C_{i}$, which represents the number of beds for department $i$. Our goal was to balance the BOR of each department; hence, our objective function is to minimize the total gap between BOR of each department and their average BOR. We developed a MIP model as follows:

(3) Objective function

$$
\min (Z)=\sum_{i=1}^{n}\left|\mathrm{BOR}_{i}\left(C_{i}\right)-\left(\sum_{i=1}^{n} \frac{\mathrm{BOR}_{i}\left(C_{i}\right)}{\mathrm{n}}\right)\right| .
$$

(4) Constraints

$$
\begin{aligned}
\mathrm{BOR}_{i}\left(C_{i}\right) & =\beta_{i}+a_{i} C_{i}+b_{i} C_{i}^{2}, \quad i=1,2, \ldots n, \\
\sum_{i=1}^{n} C_{i} & =C_{t}, \quad i=1,2, \ldots n, \\
L_{i} & \leq \mathrm{Ci} \leq U_{i}, \quad i=1,2, \ldots, n, \\
C_{i} & =K_{i 1}+2 K_{i 2}+3 K_{i 3}, \quad i=1,2, \ldots, n, \\
K_{i j} & \geq 2, \quad i=1,2, \ldots, n, j=1,2,3 .
\end{aligned}
$$

Because of the imbalance between different specialty care departments, we aimed to balance the BOR of various departments without adding extra beds. In objective function (3), $\left(\sum_{i=1}^{n} \mathrm{BOR}_{i}\left(C_{i}\right)\right) / n$ is the average BOR of $n$ departments and $Z$ is the sum of the gap between the BOR of the $n$ departments and the average of their BOR. The purpose of function (3) is to minimize the total gap in BOR between different departments and average BOR.

The constraint described by function (4) means the functional relationship between BOR and the number of beds, which is calculated in Stage II. Here, we assume that the two are quadratic functions: $\operatorname{BOR}_{i}\left(C_{i}\right)=\beta_{i}+$ $a_{i} C_{i}+b_{i} C_{i}^{2}$, where $\beta_{i}$ is a constant and $a_{i}$ and $b_{i}$ are coefficients (for more details, see Section 3.2); function (5) means that the total number of beds of the $n$ departments is constant. Function (6) ensures that there will be an upper limit and a lower limit for the beds in each department. Functions (7) and (8) impose restrictions on the ward type and patient gender. Each ward has either one, two, or three beds. The distinction between male and female wards, and the number of ward types in each department should not be less than two. For example, a single-bed ward has at least two wards so that a male patient can live in one room and a female patient can live in another single room. The other two ward types have the same conditions. Both male and female patients can decide which type of ward to live in, ensuring the fair treatment of patients.

\section{Results}

3.1. Stage I: Data Preliminaries. The LOS of 28 departments can be calculated by function (1), and the BOR of the 28 departments can be calculated by function (2). Table 2 shows the number of beds and BOR in 28 departments of the current $\mathrm{WCH}$. The BOR varies from $60.7 \%$ to $195 \%$ among the 28 departments. Some literature indicates $[32,33]$ that the optimal range for BOR is between $85 \%$ and $90 \%$. Based on this estimation, we divided these departments into three groups:

(1) Group A: BOR is less than $85 \%$. For example, W9 owns 236 beds, but its BOR is only $75.2 \%$.

(2) Group B: BOR is greater than $90 \%$. For example, the BOR of W6 reaches as high as $102 \%$, but it only has 72 beds.

(3) Group C: BOR is between $85 \%$ and $90 \%$. Their bed numbers and BOR are within the normal range, compared to groups A and B.

It is clear that there are serious imbalances in BOR between departments. To solve this problem, we interviewed a hospital manager, three other managerial assistants, and medical physicians of the ASC. We choose five departments (W9, W10, W19, W6, and W27) from groups A and B to solve the problem of bed allocation by applying the framework mentioned in Section 2.3.

3.2. Stage II: Construction of Constraint Conditions. After perprocessing the data and selecting the departments, we fitted the distribution of the patient arrival rate and LOS of the five departments using EASY-FIT, and the results are shown in Table 3.

We obtained the distribution of arrival rates for all five departments. The fitting of LOS is not ideal. Five departments do not display any distribution. We took the median of the LOS as their distribution. We used W9 as the example from which we can build the simulation model. Figure 3 describes the simulation model of W9 in Simio.

We set up a patient entity, called Patients, and a patient source called Source1, in the Simio software. We let Source1 associate with Patients and set the arrival rate to obey the Johnson distribution $(0.025,0.803,-8.16,85.98)$.

We built a Server1 to represent beds. Its Service capacity was set to the current number of beds (236), and service time 
TABle 2: Department information summary.

\begin{tabular}{|c|c|c|c|c|c|c|c|c|c|c|}
\hline \multirow{6}{*}{ A } & Department & W7 & W8 & W9 & W10 & W12 & W15 & W16 & W17 & W19 \\
\hline & Beds & 72 & 84 & 236 & 168 & 48 & 60 & 86 & 156 & 54 \\
\hline & BOR (\%) & 83.4 & 75.7 & 75.2 & 71.8 & 64.7 & 70 & 60.7 & 71.1 & 70 \\
\hline & Department & W24 & W23 & W25 & & & & & & \\
\hline & Beds & 162 & 153 & 114 & & & & & & \\
\hline & BOR (\%) & 75.60 & 84.40 & 67.60 & & & & & & \\
\hline \multirow{6}{*}{$\mathrm{B}$} & Department & W1 & W3 & W4 & W5 & W6 & W11 & W13 & W18 & W21 \\
\hline & Beds & 72 & 108 & 84 & 108 & 72 & 72 & 170 & 172 & 72 \\
\hline & BOR (\%) & 94.0 & 122 & 110 & 91 & 102 & 95.8 & 93.5 & 100 & 167 \\
\hline & Department & W26 & W27 & W26 & & & & & & \\
\hline & Beds & 91 & 114 & 91 & & & & & & \\
\hline & BOR (\%) & 195 & 137 & 195 & & & & & & \\
\hline \multirow{3}{*}{$\mathrm{C}$} & Department & W1 & W2 & W20 & W28 & & & & & \\
\hline & Beds & 84 & 66 & 72 & 114 & & & & & \\
\hline & BOR (\%) & 89.6 & 87.7 & 90 & 88.1 & & & & & \\
\hline
\end{tabular}

TABLE 3: Results of arrival rate distribution and LOS.

\begin{tabular}{lccc}
\hline Departments & Department type & Arrival rate & LOS \\
\hline W6 & B & Johnson SB $(0.289,0.983,-3.34,35.11)$ & Median $=9$ \\
W9 & A & Johnson SB $(0.025,0.803,-8.16,85.98)$ & Median $=10$ \\
W10 & A & Uniform $(-0.72,43.75)$ & Median $=12$ \\
W19 & A & Uniform $(-3.2,92.36)$ & Median $=10$ \\
W27 & B & Uniform $(-3.2,92.36)$ & Median $=5$ \\
\hline
\end{tabular}

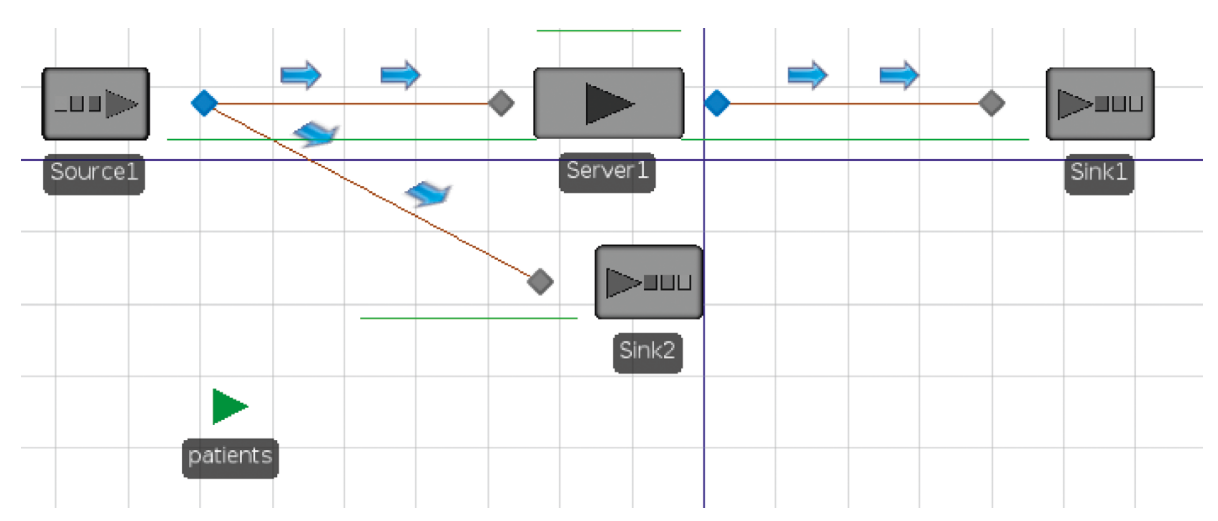

FIgURE 3: Simulation model of the W9.

was set to 10 days. We calculated the proportion of hospitalizations (43\%) based on the number of hospital admissions (12712) and discharges (5478).

There are two leaving routes in the simulation model, namely, Sink1 and Sink2. Sink1 represents the event that a patient leaves the hospital after being served by Server1. The weight from Source1 to Server 1 is $43 \%$. Sink 2 indicates that a patient who was not admitted to the hospital left the hospital directly through Sink2; the weight from Source1 to Sink2 is $57 \%$.

In order to validate the model, we run the model for a simulated year. The result was that the total number of discharges was 5984, and the BOR was $69.5 \%$. Compared with real data, the difference is $9.2 \%$ and $7.5 \%$, respectively. The error was acceptable. Then, we set different parameters for the Server1 and calculated the BOR for different scenarios.

Because W9 belongs to group A, we needed to reduce the number of beds and increase the BOR. We should therefore reduce the number of beds in Server1. In Table 4, we list the number of beds and the corresponding BOR situation for W9. The results of the other four departments are presented in Tables 5-8.

We used the number of beds as an independent variable and the BOR as the dependent variable based on the results in Table 4. The graph of BOR changing with the number of beds is shown in Figure 4. It is difficult to intuitively obtain the relationship equation between the two from the graph, so we selected eight kinds of curve functions-linear, logarithm, quadratic, composite, power, growth, exponential, 
TABLE 4: Beds and corresponding BOR of W9 in the simulation model.

\begin{tabular}{lcc}
\hline Beds & Discharge & BOR $(\%)$ \\
\hline 92 & 3266 & 97.3 \\
110 & 3896 & 97 \\
123 & 4351 & 96.9 \\
135 & 4771 & 96.8 \\
148 & 5226 & 96.7 \\
160 & 5646 & 96.68 \\
172 & 5926 & 94.4 \\
180 & 5982 & 91.1 \\
185 & 5984 & 88.6 \\
198 & 5984 & 82.8 \\
210 & 5984 & 78.1 \\
223 & 5984 & 73.5 \\
$236^{*}$ & 5984 & 69.5 \\
250 & 5984 & 65.6 \\
\hline
\end{tabular}

*The current number of hospital beds of W9.

TABLE 5: Beds and corresponding BOR of W10.

\begin{tabular}{lcc}
\hline Beds & Discharge & BOR $(\%)$ \\
\hline 68 & 2011 & 97.2 \\
78 & 2301 & 97 \\
88 & 2585 & 96.6 \\
98 & 2865 & 96.1 \\
108 & 3145 & 95.7 \\
113 & 3201 & 93.1 \\
118 & 3216 & 89.6 \\
128 & 3246 & 83.4 \\
138 & 3276 & 78 \\
148 & 3299 & 73.3 \\
158 & 3306 & 68.8 \\
168 & 3306 & 64.7 \\
178 & 3306 & 61.1 \\
\hline
\end{tabular}

TABLE 6: Beds and corresponding BOR of W6.

\begin{tabular}{lcc}
\hline Beds & Discharge & BOR $(\%)$ \\
\hline 57 & 2279 & 98.6 \\
62 & 2474 & 98.4 \\
72 & 2864 & 98.1 \\
82 & 3254 & 97.8 \\
95 & 3756 & 97.4 \\
100 & 3931 & 96.9 \\
105 & 4106 & 96.4 \\
110 & 4281 & 96 \\
115 & 4438 & 95.1 \\
120 & 4516 & 92.8 \\
125 & 4538 & 89.5 \\
130 & 4538 & 86.1 \\
140 & 4538 & 80 \\
\hline
\end{tabular}

and logistic-with which we can attempt to fit their functional relationships. We used the value of $R^{2}$ to determine which relationship function between bed and BOR of W9 had the best fit (Table 9). Since the $R^{2}$ value of the quadratic function was the best, at 0.977 , we decided that the quadratic function best describes the relationship between the bed numbers and the BOR in W9. We can derive the functional
TABLE 7: Beds and corresponding BOR of W27.

\begin{tabular}{lcc}
\hline Beds & Discharge & BOR (\%) \\
\hline 114 & 8196 & 98.50 \\
130 & 9332 & 98.30 \\
145 & 10345 & 97.70 \\
149 & 10482 & 96.37 \\
152 & 10527 & 94.90 \\
160 & 10640 & 91.10 \\
168 & 10674 & 87 \\
175 & 10681 & 83.60 \\
182 & 10688 & 80.40 \\
190 & 10696 & 77.10 \\
205 & 10711 & 71.60 \\
220 & 10726 & 66.80 \\
\hline
\end{tabular}

TABLE 8: Beds and corresponding BOR of W19.

\begin{tabular}{lcc}
\hline Beds & Discharge & BOR $(\%)$ \\
\hline 30 & 995 & 97.2 \\
35 & 1153 & 97 \\
36 & 1189 & 96.8 \\
39 & 1225 & 93 \\
38 & 1165 & 90.3 \\
40 & 1258 & 92.2 \\
45 & 1246 & 80.9 \\
47 & 1230 & 77.4 \\
50 & 1246 & 73.05 \\
52 & 1252 & 70.6 \\
54 & 1274 & 68.9 \\
\hline
\end{tabular}

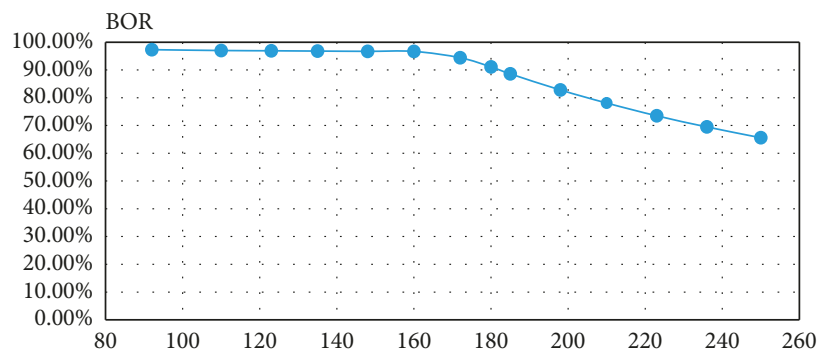

FIgURE 4: BOR curve for different numbers of beds. The abscissa is the number of beds, and the ordinate is BOR.

relationship between $\operatorname{BOR}_{1}\left(C_{1}\right)$ and the bed $C_{1}$ from Table 9; the equation is: $\mathrm{BOR}_{1}\left(C_{1}\right)=0.721+0.004 C_{1}-$ $0.0000195 C_{1}^{2}$.

Similar to the analysis process of W9, we obtained the quadratic functional relationships between the BOR and the bed number of the other four departments (Tables 10-13). Hence, the relationship function between beds and BOR of department $i$ is expressed as follows:

$$
\operatorname{BOR}_{i}\left(C_{i}\right)=\beta_{i}+a_{i} C_{i}+b_{i} C_{i}^{2},
$$

where $\mathrm{BOR}_{i}\left(C_{i}\right)$ represents the bed occupancy rate of department $i, C_{i}$ is the number of beds in department $i, \beta_{i}$ is a constant, and $a_{i}$ and $b_{i}$ are coefficients. We have obtained five equations, respectively, for five departments. They are 
TABLE 9: Relationship between bed numbers and BOR in W9.

\begin{tabular}{|c|c|c|c|c|c|c|c|c|c|}
\hline \multirow{2}{*}{ Function } & \multicolumn{5}{|c|}{ Model } & \multicolumn{4}{|c|}{ Parameters } \\
\hline & $R^{2}$ & $\mathrm{~F}$ & df1 & $\mathrm{df} 2$ & Sig. & Constant & b1 & b2 & b3 \\
\hline Linear & 0.836 & 61.326 & 1 & 12 & 0.000 & 1.253 & -0.002 & & \\
\hline Logarithm & 0.724 & 31.538 & 1 & 12 & 0.000 & 2.550 & -0.328 & & \\
\hline Quadratic & 0.977 & 230.860 & 2 & 11 & 0.000 & 0.721 & 0.004 & 0.0000195 & \\
\hline Composite & 0.820 & 54.831 & 1 & 12 & 0.000 & 1.367 & 0.997 & & \\
\hline Power & 0.704 & 28.548 & 1 & 12 & 0.000 & 6.455 & -0.392 & & \\
\hline Growth & 0.820 & 54.831 & 1 & 12 & 0.000 & 0.313 & -0.003 & & \\
\hline Exponential & 0.820 & 54.831 & 1 & 12 & 0.000 & 1.367 & -0.003 & & \\
\hline Logistic & 0.820 & 54.831 & 1 & 12 & 0.000 & 0.732 & 1.003 & & \\
\hline
\end{tabular}

TABLE 10: Relationship between bed numbers and BOR in W10.

\begin{tabular}{|c|c|c|c|c|c|c|c|c|c|}
\hline \multirow{2}{*}{ Function } & \multicolumn{5}{|c|}{ Model } & \multicolumn{4}{|c|}{ Parameters } \\
\hline & $R^{2}$ & $\mathrm{~F}$ & df1 & $\mathrm{df} 2$ & Sig. & Constant & b1 & b2 & b3 \\
\hline Linear & 0.919 & 125.130 & 1 & 11 & 0.000 & 1.298 & -0.004 & & \\
\hline Logarithm & 0.837 & 56.598 & 1 & 11 & 0.000 & 2.813 & -0.413 & & \\
\hline Quadratic & 0.973 & 181.654 & 2 & 10 & 0.000 & 0.895 & 0.003 & $-2.854 E-5$ & \\
\hline Composite & 0.907 & 107.562 & 1 & 11 & 0.000 & 1.468 & 0.995 & & \\
\hline Power & 0.817 & 49.021 & 1 & 11 & 0.000 & 9.578 & -0.513 & & \\
\hline Growth & 0.907 & 107.562 & 1 & 11 & 0.000 & 0.384 & -0.005 & & \\
\hline Exponential & 0.907 & 107.562 & 1 & 11 & 0.000 & 1.468 & -0.005 & & \\
\hline Logistic & 0.907 & 107.562 & 1 & 11 & 0.000 & 0.681 & 1.005 & & \\
\hline
\end{tabular}

Note. The higher the $R^{2}$, the better the function model. In the W10 model fitting, the fitting degree of the quadratic curve is the best, and the quadratic curve is directly selected. The bed number of W10 is $C_{2}$, and the bed utilization rate is $\mathrm{BOR}_{2}\left(C_{2}\right)$. According to the estimated value of the parameter, $\operatorname{BOR}_{2}\left(C_{2}\right)=0.895+0.003 C_{2}-0.00002854 C_{2}^{2}$.

TABLE 11: Relationship between beds and BOR in W6.

\begin{tabular}{|c|c|c|c|c|c|c|c|c|c|}
\hline \multirow{2}{*}{ Function } & \multicolumn{5}{|c|}{ Model } & \multicolumn{4}{|c|}{ Parameters } \\
\hline & $R^{2}$ & $\mathrm{~F}$ & df1 & $\mathrm{df} 2$ & Sig. & Constant & b1 & $\mathrm{b} 2$ & b3 \\
\hline Linear & 0.664 & 21.741 & 1 & 11 & 0.001 & 1.118 & -0.002 & & \\
\hline Logarithm & 0.558 & 13.883 & 1 & 11 & 0.003 & 1.616 & -0.147 & & \\
\hline Quadratic & 0.956 & 107.465 & 2 & 10 & 0.000 & 0.687 & 0.008 & $-4.932 E-5$ & \\
\hline Composite & 0.645 & 19.978 & 1 & 11 & 0.001 & 1.140 & 0.998 & & \\
\hline Power & 0.539 & 12.849 & 1 & 11 & 0.004 & 1.966 & -0.161 & & \\
\hline Growth & 0.645 & 19.978 & 1 & 11 & 0.001 & 0.131 & -0.002 & & \\
\hline Exponential & 0.645 & 19.978 & 1 & 11 & 0.001 & 1.140 & -0.002 & & \\
\hline Logistic & 0.645 & 19.978 & 1 & 11 & 0.001 & 0.877 & 1.002 & & \\
\hline
\end{tabular}

Note. The higher the $R^{2}$, the better the function model. When the W6 model is fitted, it has the same outcome as W9. The quadratic curve is also selected. The bed number of W6 is $C_{3}$, and the bed rate is $\mathrm{BOR}_{3}\left(C_{3}\right)$. According to the estimated value of the parameter, $\mathrm{BOR}_{3}\left(C_{3}\right)=0.687+0.008 C_{3}-0.000049323 C_{3}^{2}$.

TABLE 12: Relationship between beds and BOR in W27.

\begin{tabular}{lcccccccc}
\hline \multirow{2}{*}{ Function } & \multicolumn{3}{c}{ Model } & \multicolumn{3}{c}{ Parameters } \\
& $R^{2}$ & $\mathrm{~F}$ & $\mathrm{df1}$ & $\mathrm{df2}$ & Sig. & Constant & b1 & b2 \\
\hline Linear & 0.932 & 137.447 & 1 & 10 & 0.000 & 1.446 & -0.003 & \\
Logarithm & 0.882 & 74.410 & 1 & 10 & 0.000 & 3.673 & -0.550 & $-2.007 E-5$ \\
Quadratic & 0.967 & 131.496 & 2 & 9 & 0.000 & 0.901 & 0.003 & $-2.585 E-6$ \\
Composite & 0.964 & 119.131 & 2 & 9 & 0.000 & 1.094 & 0.000 & $-3.039 E-8$ \\
Power & 0.924 & 121.995 & 1 & 10 & 0.000 & 1.720 & 0.996 & -0.655 \\
Growth & 0.866 & 64.725 & 1 & 10 & 0.000 & 24.281 & -004 & \\
Exponential & 0.924 & 121.995 & 1 & 10 & 0.000 & 0.542 & -0.004 & \\
Logistic & 0.924 & 121.995 & 1 & 10 & 0.000 & 1.720 & -0.004 & \\
\hline
\end{tabular}

Note. The higher the $R^{2}$, the better the function model. In the W27 model fitting, the fitting of the quadratic curve is the best, and the quadratic curve is selected. The bed number of W27 is $C_{4}$, and the bed utilization rate is $\mathrm{BOR}_{4}\left(C_{4}\right)$. According to the estimated value of the parameter, $\mathrm{BOR}_{4}\left(C_{4}\right)=0.901+0.003 C_{4}-0.000020066 C_{4}^{2}$. 
TABLE 13: Relationship between beds and BOR in W19.

\begin{tabular}{|c|c|c|c|c|c|c|c|c|c|}
\hline \multirow{2}{*}{ Function } & \multicolumn{5}{|c|}{ Model } & \multicolumn{4}{|c|}{ Parameters } \\
\hline & $R^{2}$ & $\mathrm{~F}$ & df1 & $\mathrm{df} 2$ & Sig. & Constant & b1 & b2 & b3 \\
\hline Linear & 0.947 & 159.652 & 1 & 9 & 0.000 & 1.450 & -0.014 & & \\
\hline Logarithm & 0.915 & 96.619 & 1 & 9 & 0.000 & 3.011 & -0.579 & & \\
\hline Quadratic & 0.966 & 114.721 & 2 & 8 & 0.000 & 0.911 & 0.012 & $-3.054 E-5$ & \\
\hline Composite & 0.964 & 108.277 & 2 & 8 & 0.000 & 1.095 & 0.000 & $-5.576 E-5$ & $-1.687 E-6$ \\
\hline Power & 0.944 & 151.827 & 1 & 9 & 0.000 & 1.736 & 0.983 & & \\
\hline Growth & 0.908 & 88.465 & 1 & 9 & 0.000 & 11.291 & -0.695 & & \\
\hline Exponential & 0.944 & 151.827 & 1 & 9 & 0.000 & 0.552 & -0.017 & & \\
\hline Logistic & 0.944 & 151.827 & 1 & 9 & 0.000 & 1.736 & -0.017 & & \\
\hline
\end{tabular}

Note. The higher the $R^{2}$, the better the function model. In the W19 model fitting, the fitting of the quadratic curve is the best, and the quadratic curve is selected. The bed number of $\mathrm{W} 19$ is $C_{5}$, and the bed utilization rate is $\mathrm{BOR}_{5}\left(C_{5}\right)$. According to the estimated value of the parameter, $\mathrm{BOR}_{5}\left(C_{5}\right)=0.911+0.012 C_{5}-0.0003054 C_{5}^{2}$

used as constraints for the mixed integer programming model in Stage III.

\subsection{Stage III: A Mixed Integer Programming Model. After} data analysis and simulation in the first two stages, we obtained the parameter values in equations (3)-(8), including the five selected departments and established the quadratic function relationship between the bed numbers and the BOR. We then applied these parameters to equations (3)-(8) to solve the model. The specific MIP model is as follows:

Objective function

$$
\min (Z)=\sum_{i=1}^{5}\left|\mathrm{BOR}_{i}\left(C_{i}\right)-\left(\sum_{i=1}^{5} \frac{\mathrm{BOR}_{i}\left(C_{i}\right)}{5}\right)\right| .
$$

\section{Constraints}

$$
\begin{aligned}
\mathrm{BOR}_{1}\left(C_{1}\right) & =0.721+0.004 C_{1}-0.0000195 C_{1}^{2}, \\
\mathrm{BOR}_{2}\left(C_{2}\right) & =0.895+0.003 C_{2}-0.00002854 C_{2}^{2}, \\
\mathrm{BOR}_{3}\left(C_{3}\right) & =0.687+0.008 C_{3}-0.000049323 C_{3}^{2}, \\
\mathrm{BOR}_{4}\left(C_{4}\right) & =0.901+0.003 C_{4}-0.000020066 C_{4}^{2}, \\
\mathrm{BOR}_{5}\left(C_{5}\right) & =0.911+0.012 C_{5}-0.0003054 C_{5}^{2}, \\
\sum_{i=1}^{5} C_{i} & =644, \\
92 & \leq C_{1} \leq 210, \\
68 & \leq C_{2} \leq 138, \\
57 & \leq C_{3} \leq 140,
\end{aligned}
$$

$$
\begin{aligned}
& 114 \leq C_{4} \leq 182, \\
& 30 \leq C_{5} \leq 182, \\
& C_{i}=K_{i 1}+2 K_{i 2}+3 K_{i 3}, \quad i=1,2,3,4,5, \\
& K_{i j} \geq 2, \quad i=1,2,3,4,5, j=1,2,3 .
\end{aligned}
$$

Constraints (11)-(15) are quadratic functions of the number of beds and the BOR of the five departments. After we obtain the number of beds in each department, we can calculate their BOR by formulas (11)-(15). Constraint (16) states the total number of beds in five departments. Constraints (17)-(21) limit the upper bound and lower bound on bed numbers of each department. Constraints (22) and (23) restrict the ward type and patient gender. There are three ward types for each department in WCH. So, the number of beds in department $i$ is the sum of the total number of beds from those three types. In order to distinguish the male and female wards, the number of each department type must be a positive integer and should not be less than two. We used the genetic algorithm [34] to solve the MIP model. The genetic algorithm is run on a personal computer with an Intel ${ }^{\circledR}$ Core $^{\mathrm{TM}}$ i7-7700 CPU, a $3.60 \mathrm{GHz}$ z Intel processor, and 8.0 GB RAM. The elapsed time is 77.652611 seconds.

We analyzed the results from three aspects:

(1) Initial bed allocations and optimal bed allocations based on our model: as shown in Figure 5, the blue histograms represent the Initial bed allocation, which is the current hospital bed number. The optimal bed allocations from our model are represented by the yellow histograms. Figure 5 shows the optimal bed allocation strategy: the bed of the W9 reduces from 236 to 166 , W10 from 168 to 121, and W19 from 54 to 44; W6 increases from 72 to 135 and W27 from 114 to 178 .

(2) Initial BOR and optimal BOR: Figure 6 shows the corresponding BOR after optimization. The W9 increases from $69.5 \%$ to $84.76 \%$, W 10 from $64.7 \%$ to $84.01 \%$, W 19 from $68.9 \%$ to $84.78 \%$; on the contrary, W6 decreases from $98.1 \%$ to $86.81 \%$ and W27 from $98.5 \%$ to $83.48 \%$. Blue lines represent the original 


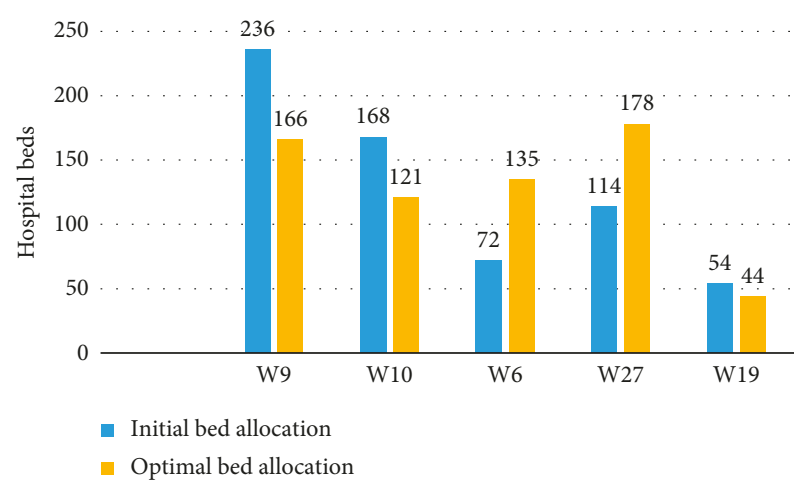

FIGURE 5: Initial bed allocation strategy and optimal bed allocation strategy.

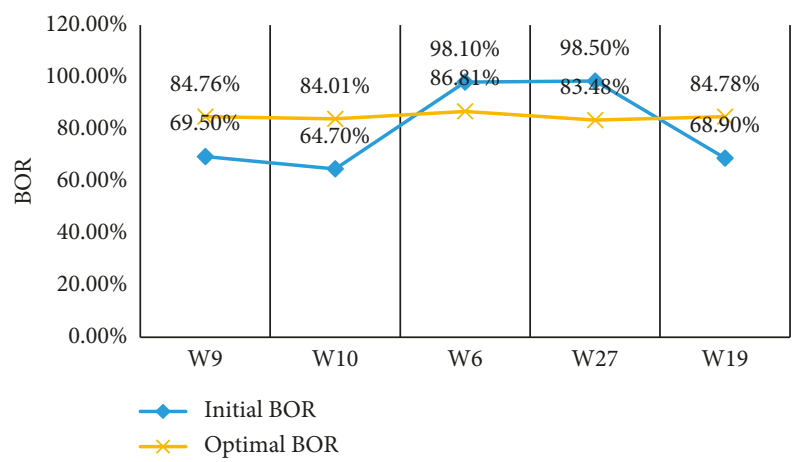

FIgURE 6: Results of initial BOR and optimal BOR.

BOR, and yellow lines represent the optimal BOR. We found that the maximum and minimum BORs are $98.1 \%$ and $64.7 \%$. The maximum difference of BOR is $33.8 \%$ but change to $2.80 \%$ after optimization.

(3) Objective function value: our objective value, $Z$, represents the imbalance degree of bed utilization between various departments. For baseline bed allocation, the initial value of the objective function $Z$ is as high as 0.7344 . After optimization, the optimal value of $Z$ is 0.0409 , indicating that our optimization reduced the severity of the imbalance.

Finally, we can get a combination of beds in different wards based on the number of optimal beds (Table 14). In formula (22), $C_{i}$ depends on the value of $K_{i j}$, that is to say, the combinations of $K_{i j}$ produce different $C_{i}$ values. For example, the optimal number of beds for W9 determined by our model is $166\left(C_{1}=166\right)$; there are many combinations for single-bed wards $\left(K_{11}\right)$, double-bed wards $\left(K_{12}\right)$, and triple-bed wards $\left(K_{13}\right)$, such as $(26,40,20),(22,42,20)$, and $(36,20,30)$. This means that W9 can provide 26 single-bed wards, 40 double-bed wards, and 20 triple-bed wards; 22 single-bed wards, 42 double-bed wards, and 20 triple-bed wards; or 36 single-bed wards, 20 double-bed wards, and 30 triple-bed wards; and so on.

\section{Discussion}

Many tertiary hospitals in China are facing the same problem as WCH, with respect to the imbalance in the
TABLE 14: Optimal number of beds and different combinations of departments.

\begin{tabular}{lcc}
\hline Department & $\begin{array}{c}\text { Optimal } \\
\text { number of beds }\end{array}$ & $\begin{array}{c}\text { Different feasible combinations of } \\
K_{i j}:\left(K_{i 1}, K_{i 2}, K_{i 3}\right)\end{array}$ \\
\hline W9 & 166 & $\left(K_{11}, K_{12}, K_{13}\right):(26,40,20),(22$, \\
$42,20),(36,20,30), \ldots$ \\
$\left(K_{21}, K_{22}, K_{23}\right):(21,20,20),(21$, \\
W10 & $35,10),(31,30,10), \ldots$ \\
& 121 & $\left(K_{31}, K_{32}, K_{33}\right):(35,20,20),(20$, \\
W6 & $40,7),(25,10,30), \ldots$ \\
W27 & \multirow{3}{*}{135} & $\left(K_{41}, K_{42}, K_{43}\right):(78,20,20),(10$, \\
& $39,30),(40,39,20), \ldots$ \\
W19 & \multirow{3}{*}{44} & $\left(K_{51}, K_{52}, K_{53}\right):(14,6,6),(4,5$, \\
& & $10),(19,5,5), \ldots$ \\
\hline
\end{tabular}

utilization of bed resources in different departments. The availability of beds for hospital care is excessive in some cases and scarce in others. This phenomenon has caused many problems for hospitals. For example, some hospital wards are always overcrowded, while others are underloaded. Some scheduled patient admissions are delayed or even transferred to other hospitals, and some patients are hospitalized in inappropriate wards which are unsuited to their pathologies, with the risk of a lower quality care and a greater chance of infection [35].

To relieve this imbalance, we propose a data-driven hybrid three-stage framework combining multiple methods to produce a feasible bed allocation strategy since it is difficult to allocate beds among all 28 departments in the whole WCH. We selected five departments (W9, W10, W6, W27, and W19) through data analysis and survey interview. W9, W10, and W19 are departments that have many beds with a low BOR while W6 and W27 have few beds with high BOR. For Stage II, we developed a generic discrete-event simulation model. We fitted the relationship function between BOR and beds of each wards via the simulation model. In Stage III, we developed a MIP model to minimize the imbalance in BOR. The results of Stage II are incorporated into the MIP model as one of the key constraints. We also considered other constraints, such as ward types (single, double, and three-bed wards) and upper and lower bounds on the number of beds.

Our data-driven hybrid three-stage framework produces a flexible allocation strategy for hospital bed management. Our research helps to improve the utilization of medical resources and the quality of medical services by balancing bed numbers and BOR between different departments. Our model may be applied in two ways. Firstly, it can be extended to other wards with different arrival rates and LOS distribution. Secondly, our study can provide a reference for dealing with the problem of hospital bed capacity to other large general hospitals in China. Our research provides a common framework for hospital bed allocation, so other departments or hospitals can follow our three-stages framework to realize their allocation of beds. Because the data of each hospital and the actual situation are different from those of $\mathrm{WCH}$, different constraints or objective functions may be generated. For example, other hospitals 
can use data analysis to screen the departments that need to allocate beds; then, they can follow the method described in Stage II to fit the functional relationship between BOR and bed. Different hospitals may have different functional relationships because of their different data. Finally, hospitals can redesign the model with more personalized objective functions and constraints according to their own actual situation.

\section{Conclusions}

We focused upon the problem of allocating beds among different departments in a hospital. We took a large public hospital in China, WCH, as a case study. To relieve imbalances in BOR between departments, we proposed a threestage framework. In the first stage, we collected data and identified departments of interest. In the second stage, we identified the functional relationship between the number of beds and the BOR. The third-stage MIP model provides the best number of bed allocations for different departments. It has proven to be a feasible method to ease the shortage of beds.

Our research is based on real data, and hospital managers can draw upon the results of this study to solve the bed occupancy and capacity problem. The three-stage framework can help bed managers adjust the allocation of beds in a timely and dynamic manner. This approach can be applied to the majority of other hospitals and may serve as a starting point for the development of allocation models for other service industries with similar conditions, such as the allocation of beds or room types in hotels.

Future study can consider the following two aspects: since this is the initial stage of bed allocation, the strategy can be extended to more departments. In addition, the practice will be a good reference for other large general hospitals in China. More factors may be considered for inclusion in the MIP model such as other ward resources (nurses and doctors), infectious patients, and the undesirability of mixed-sex rooms.

\section{Appendix}

General hospitals have been divided into three levels in China, according to the hospital's functions, tasks, facilities, technology, medical services, and scientific management.

Class I hospitals (the number of beds is 100 or fewer): primary hospitals and health centers that provide prevention, medical care, health care, and rehabilitation services directly to communities in a specific population.

Class II hospitals (101 to 500 beds): regional hospitals that provide comprehensive medical and health services to multiple communities and undertake certain teaching and research tasks.

Class III hospitals (also called tertiary hospitals; authors' hospital; more than 501 beds): regional or higher hospitals that provide high-level specialized medical and health services and perform higher education and scientific research tasks in several areas. Tertiary hospitals are further subdivided into three grades based upon the technical strength of the hospital, management levels, equipment conditions, scientific research capabilities, and more. The West China Hospital is one of the highest-grade hospitals among the tertiary hospitals.

\section{Data Availability}

The data used to support the findings of this study are restricted by the West China Hospital in order to protect patient privacy. Data are available from West China Hospital for researchers who meet the criteria for access to confidential data.

\section{Conflicts of Interest}

The authors declare that there are no conflicts of interest regarding the publication of this paper.

\section{Acknowledgments}

The authors gratefully acknowledge the support from the Admission Service Center of West China Hospital. This research was supported in part by the National Natural Science Foundation of China (Nos. 71532007 and 71131006) and Sichuan Province Science and Technology Support Project Plan (No. 2016FZ0080).

\section{References}

[1] World Health Organization, Tracking Universal Health Coverage: First Global Monitoring Report, World Health Organization, Geneva, Switzerland, 2015, http://www.who. int/healthinfo/universal_health_coverage/report/2015/en/.

[2] Beijing: China Union Medical University Press. 2016, http:// www.nhfpc.gov.cn/guihuaxxs/s10748/201708/d82fa7141696407 abb4ef764f3edf095.shtml?from=groupmessage\&isappinstalled $=1$.

[3] T. J. Best, Hospital Bed Capacity Management, Dissertations \& Theses-Gradworks, Chicago, IL, USA, June 2015.

[4] D. J. Worthington, "Queueing models for hospital waiting lists," Journal of the Operational Research Society, vol. 38, no. 5, pp. 413-422, 1987.

[5] R. Akkerman and M. Knip, "Reallocation of beds to reduce waiting time for cardiac surgery," Health Care Management Science, vol. 7, no. 2, pp. 119-126, 2004.

[6] K. Seung-Chul, H. Ira, Y. Karl K, and B. Thomas A, "Flexible bed allocation and performance in the intensive care unit," Journal of Operations Management, vol. 18, no. 4, pp. 427-443, 2000.

[7] D. Kleinloog, F. B. Houckgeest, and D. Sierink, "Triage after drug overdose: effect of the introduction of a medical psychiatry unit on the allocation of ICU beds," Critical Care, vol. 19, no. 1, p. P513, 2015.

[8] L. V. Green, "How many hospital beds?," British Medical Journal, vol. 39, pp. 400-412, 2003.

[9] A. J. M. Oerlemans, H. Wollersheim, N. V. Sluisveld, J. G. V. D. Hoeven, W. J. M. Dekkers, and M. Zegers, "Rationing in the intensive care unit in case of full bed occupancy: a survey among intensive care unit physicians," BMC Anesthesiology, vol. 16, no. 1, p. 25, 2015.

[10] P. Devapriya, C. T. Strömblad, M. D Bailey et al., "StratBAM: a discrete-event simulation model to support strategic hospital bed capacity decisions," Journal of Medical Systems, vol. 39, no. 10, p. 130, 2015. 
[11] J. C. Ridge, S. K. Jones, M. S. Nielsen, and A. K. Shahani, "Capacity planning for intensive care units," European Journal of Operational Research, vol. 105, no. 2, pp. 346-355, 1998.

[12] G. Romanin-Jacur and P. Facchin, "Optimal planning of a pediatric semi-intensive care unit via simulation," European Journal of Operational Research, vol. 29, no. 2, pp. 192-198, 1987.

[13] E. Akcali, M. J. Côté, and C. Lin, "A network flow approach to optimizing hospital bed capacity decisions," Health Care Management Science, vol. 9, no. 4, pp. 391-404, 2006.

[14] M. Utley, S. Gallivan, T. Treasure, and O. Valencia, "Analytical methods for calculating the capacity required to operate an effective booked admissions policy for elective inpatient services," Health Care Management Science, vol. 6, no. 2, pp. 97-104, 2003.

[15] C. Lakshmi and S. A. Iyer, "Application of queueing theory in health care: a literature review," Operations Research for Health Care, vol. 2, no. 1-2, pp. 25-39, 2013.

[16] U. Uzunoglu Kocer and S. Ozkar, " $\mathrm{M} / \mathrm{C}_{2} / \mathrm{c} / \mathrm{K}$ queuing model and optimization for a geriatric care center," Applied Stochastic Models in Business and Industry, vol. 31, no. 6, pp. 892-911, 2015.

[17] S. Belciug and F. Gorunescu, "A hybrid genetic algorithmqueuing multi-compartment model for optimizing inpatient bed occupancy and associated costs," Artificial Intelligence in Medicine, vol. 68, pp. 59-69, 2016.

[18] T. P. Roh, T. R. Huschka, M. J. Brown, and Y. N. Marmor, "Data-driven simulation use to determine bed resource requirements for the redesign of pre- and post-operative care areas," in Proceedings of the Winter Simulation Conference, vol. 1, pp. 1168-1176, Washington, DC, USA, 2014.

[19] E. W. Hans and A. G. Leeftink, "Integral planning of operating rooms and wards: tactical allocation of beds to reduce fluctuations in bed utilization for Medisch Spectrum Twente," in Enschede, F. C. Smit, Ed., pp. 1-114, General Books LLC, Memphis, TN, USA, 2015.

[20] J. T. Blake and M. W. Carter, "A goal programming approach to strategic resource allocation in acute care hospitals," European Journal of Operational Research, vol. 140, no. 3, pp. 541-561, 2002.

[21] M. M. Hossein, K. Sajad, and T. Mahya, "Reallocation of shafa hospital beds in kerman using goal programming model," Electronic Physician, vol. 8, no. 8, pp. 2733-2737, 2016.

[22] I. Marques, M. E. Captivo, and M. Vaz Pato, "An integer programming approach to elective surgery scheduling," $O R$ Spectrum, vol. 34, no. 2, pp. 407-427, 2012.

[23] S. Y. Shin, Y. Brun, H. Balasubramanian, P. L. Henneman, and L. J. Osterweil, "Discrete-event simulation and integer linear programming for constraint-aware resource scheduling," IEEE Transactions on Systems, Man, and Cybernetics: Systems, vol. 48, no. 9, pp. 1578-1593, 2018.

[24] R. B. Bachouch, A. Guinet, and S. Hajri-Gabouj, "An integer linear model for hospital bed planning," International Journal of Production Economics, vol. 140, no. 2, pp. 833-843, 2012.

[25] C. Tontarski, "Modeling and analysis of OR nurse scheduling using mathematical programming and simulation," Dissertations \& Theses-Gradworks, 2015.

[26] E. P. C. Kao and G. G. Tung, "Bed allocation in a public health care delivery system," Management Science, vol. 27, no. 5, pp. 507-520, 1981.

[27] M. B. Dumas, "Simulation modeling for hospital bed planning," Simulation, vol. 43, no. 2, pp. 69-78, 1984.
[28] J. A. Joines and S. D. Roberts, Simulation Modeling with Simio: A Workbook, Simio LLC, Sewickley, PA, USA, 2013.

[29] Q. Xiao, L. Luo, S.-Z. Zhao, X.-B. Ran, and Y.-B. Feng, "Online appointment scheduling for a nuclear medicine department in a Chinese hospital," Computational and Mathematical Methods in Medicine, vol. 2018, no. 10, pp. 1-13, 2018.

[30] L. B. Holm, H. Lurås, and F. A. Dahl, "Improving hospital bed utilisation through simulation and optimisation: with application to a $40 \%$ increase in patient volume in a Norwegian general hospital," International Journal of Medical Informatics, vol. 82, no. 2, pp. 80-89, 2013.

[31] K. Schittkowski, "EASY-FIT: a software system for data fitting in dynamical systems," Structural and Multidisciplinary Optimization, vol. 23, no. 2, pp. 153-169, 2002.

[32] S. Belciug and F. Gorunescu, "Improving hospital bed occupancy and resource utilization through queuing modeling and evolutionary computation," Journal of Biomedical Informatics, vol. 53, pp. 261-269, 2015.

[33] J. K. Cochran and A. Bharti, "Stochastic bed balancing of an obstetrics hospital," Health Care Management Science, vol. 9, no. 1, pp. 31-45, 2006.

[34] D. Whitley, "A genetic algorithm tutorial," Statistics and Computing, vol. 4, no. 2, pp. 65-85, 1994.

[35] J. Orendi, "Health-care organisation, hospital-bed occupancy, and MRSA," The Lancet, vol. 371, no. 9622, pp. 1401-1402, 2008. 


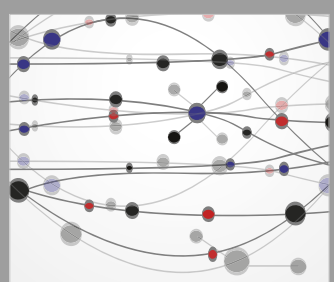

The Scientific World Journal
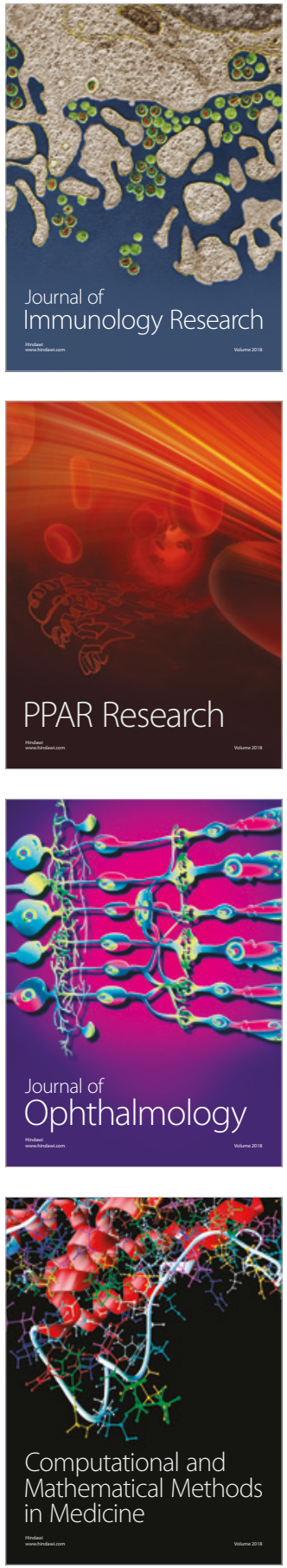

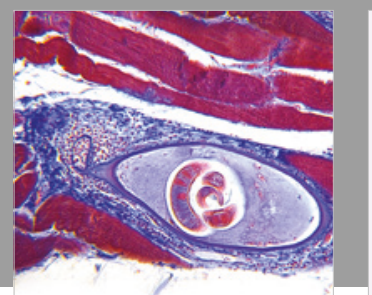

Gastroenterology Research and Practice

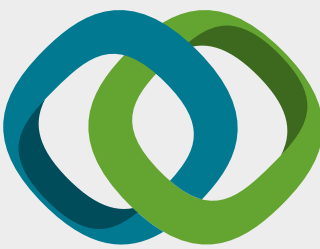

\section{Hindawi}

Submit your manuscripts at

www.hindawi.com
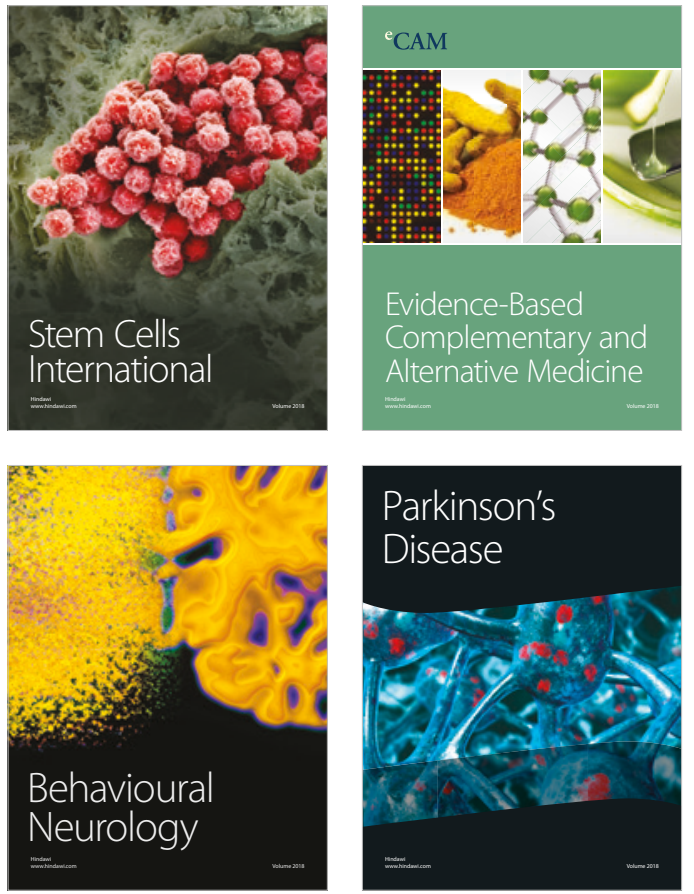

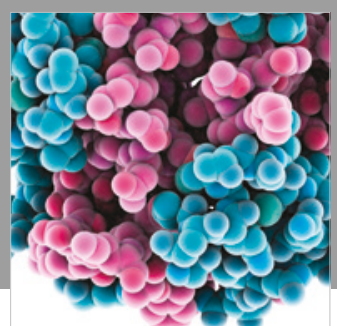

ournal of

Diabetes Research

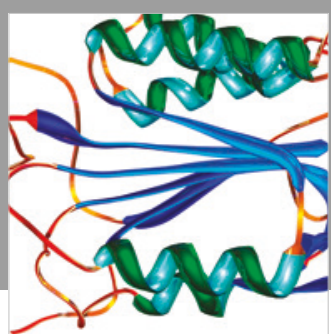

Disease Markers
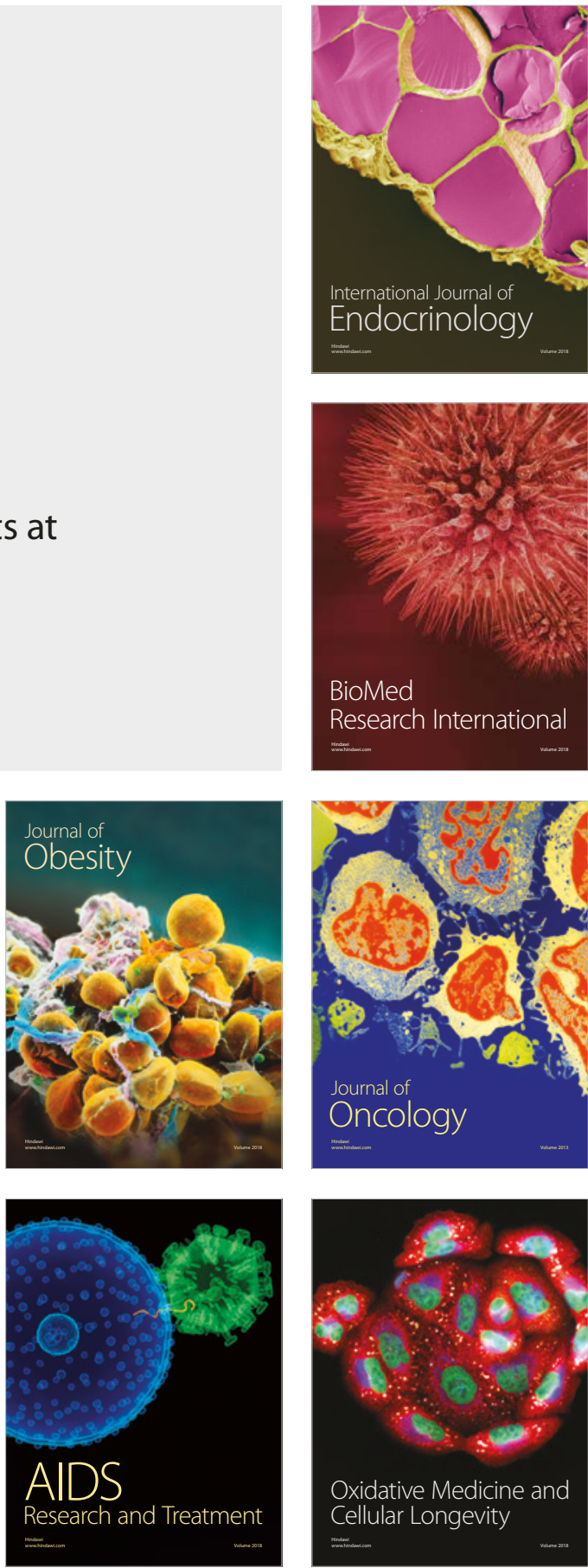